\title{
IMPACT OF MATERNAL NUTRITION ON CHILD HEALTH
}

\author{
Naushaba Akhtar ${ }^{1}$, Subhadarshini Satapathy ${ }^{2}$
}

${ }^{1}$ Post Graduate Student, Asian Institute of Public Health, Pahala, Bhubaneswar, Odisha, India

${ }^{2}$ Clinical Dietician, HCG Panda Cancer Hospital, Telengapentha, Cuttack, Odisha, India

Article Info: Received 05 January 2021; Accepted 18 February 2021

DOI: https://doi.org/10.32553/jbpr.v10i2.854

Corresponding author: Naushaba Akhtar

Conflict of interest statement: No conflict of interest

\section{Abstract}

Background: The state of under nutrition among women in India has remained a prevalent cause for affecting maternal and child health and increasing neonatal deaths.

Objective: In this paper, the authors have reviewed and summarized the complete knowledge of maternal nutrition requirements, how maternal nutrition impacts child health and public health considerations.

Method: Review of existing scientific literature and records from the past.

Conclusion: In this article, the author concludes that nutrition is one of those factors which can be easily modified and it is of great public health importance, giving importance to nutrition can be of great health to mother and child and reduce birth complications and adverse birth outcomes.

Keywords: Nutrition, Maternal health, Maternal nutrition, Child Health, Birth outcomes.

\section{Introduction}

Nutrition has always been a vital component of all living things. The importance of nutrition in the diet is increasing as we are moving towards modernization and globalization. The changing environment and requires changes and manipulation in nutrition intake. The nutritional value of adult women is of great importance and plays a vital role in the development of a child. ${ }^{1}$ The nutrition consumed by the mother directly impacts the foetus hence impacts birth outcomes, neonatal and child health. ${ }^{2}$ There have numerous studies conducted on the issue of maternal nutrition and birth outcomes, which shows maternal inadequate nutrition may cause low birth weights, intrauterine growth retardation, preterm birth, other birth outcomes. Some studies have investigated the impact of single nutrition on maternal and child health (e.g. Iodine, Iron, various Vitamins \& minerals). ${ }^{3}$
It has been evaluated in numerous researches that there are secondary factors responsible for assessing the nutritional status. The nutritional status of a person depends upon the following factors: (i) Demographical: age, gender, geographical location, (ii) biological: growth\& development and other co-morbidity (if any), (iii) socio-economical: lifestyle, Occupation (both duration \& type), income, knowledge and environment. Some studies have also identified that nutrition deficiency is mostly seen in a population of Low middle-income nations and nutrition has remained one of the very important factors for neonatal deaths. ${ }^{4}$

Some studies have shown the importance of findings of child-health problem related to specific nutrient. This paper focuses on categorizing the importance of nutritional requirement for an adult woman, aged 1849years, and identifies its impacts on Child health. In this paper, we have categorized all 
the required nutrients necessary for maternal health, especially during pregnancy, during the second and third trimester, which is considered the most important stage of pregnancy. ${ }^{5}$ The table below shows the nutrients and amount required for an adult pregnant woman. This multi-nutrient table tries to bring about a broader understanding of specific essential nutrients and it's required amount for a pregnant woman. These include energy, protein, essential fatty acids (omega-3), iron, magnesium, zinc, calcium and other vitamins. ${ }^{6}$

Table 1: Shows selected nutrients requirement for an adult pregnant woman (for $2^{\text {nd }} \& 3^{\text {rd }}$ trimester). Source: ICMR recommended dietary allowances for an expectant mother-2010

\begin{tabular}{|l|l|}
\hline NUTRIENT & AMOUNT \\
\hline Energy & $2580 \mathrm{kcal}$ \\
\hline Protein & $78 \mathrm{~g}$ \\
\hline Fat & $30 \mathrm{~g}$ \\
\hline Calcium & $1200 \mathrm{mg}$ \\
\hline Iron & $35 \mathrm{mg}$ \\
\hline Vitamin C & $60 \mathrm{mg}$ \\
\hline Magnesium & $310 \mathrm{mg}$ \\
\hline Zinc & $12 \mathrm{mg}$ \\
\hline
\end{tabular}

Maternal Nutrition \& Child Health

Maternal nutrition is important during pregnancy as a mother is the only source of nutrition for the child and the food eaten by the mother affects child growth and birth outcomes. ${ }^{7}$ The improper or inadequate intake of nutrition can give rise to various birth complications and thereby affecting child growth and development. The impact of nutrition on child health during birth are seen as the adverse outcomes observed right after birth and which can also have serious long-term consequences on health and even death. ${ }^{8}$ The adverse birth outcomes mainly are: low birth weight, intrauterine growth retardation and preterm birth and child growth affect observed are stunting and long-term cognitive impact. ${ }^{9}$

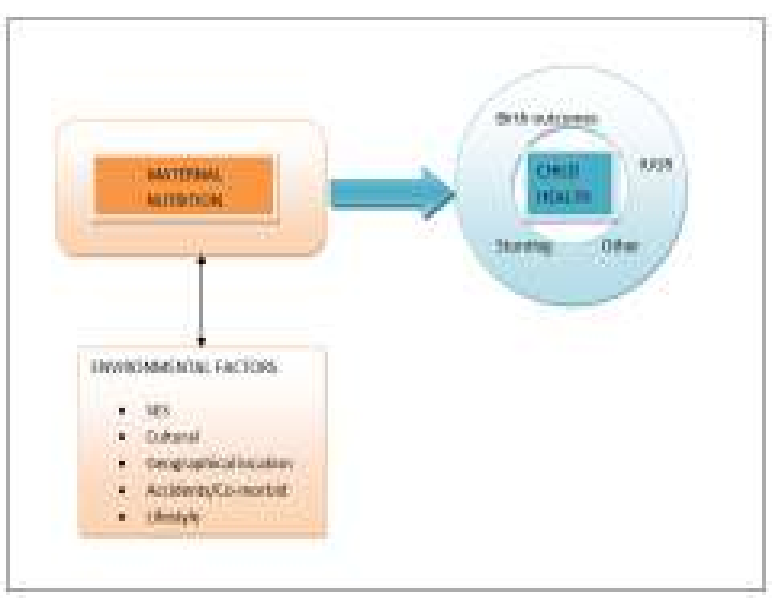

Figure 1: Shows the association between maternal nutrition and child health. Author's conceptualization

Figure 1 shows the association between maternal nutrition and child health, thereby leading to birth outcomes and other issues like stunting, IUGR, this model also shows the role of environmental factors in association with maternal nutrition status. The associated factors include:

SES: The socio-economic status of the population, education and knowledge of the mother adversely affect the intake of nutrition and thereby indirectly affecting child health. ${ }^{14}$ Culture: The culture of the region also affects nutrition and diet. Geographical location: The geography of the region determines the availability of adequate resources in the region, such as Low income and Low middle-income nations have compromised nutritional status. Accidents/comorbidity: These are conditions that cause distress and impairment which also affects nutrition intake and ultimately health. Lifestyle: This includes work style, pattern, occupation, living and other built environment which can be responsible for the accessibility of nutrition both financial and geographical. The social inequality usually includes income status, type of occupation, attainment of education and a good level of education has remained one of the key factors for attainment of good health and is one of the consistent socioeconomic predictors. At the societal level, such factors act as "upstream" factors, whereas maternal nutrition here acts as a "downstream" factor or 
"individual" level factor. The low middleincome countries have been showing poor maternal nutrition level and increased number of birth outcomes, more number of postnatal complication and even an increase in neonatal deaths. It has been evident that the majority of neonatal deaths results during the first week of life and nearly 1 million newborn deaths occur within the first 24 hours of their life. The common conditions observed among them include Preterm birth, Intrapartum-related complication, infections (due to compromised immune system) and birth defects. ${ }^{10}$ These adverse outcomes have been identified as the leading causes of neonatal mortality. ${ }^{11}$ These birth outcomes also give rise to short-term and long-term health consequences, short-term include some health problems and disabilities and long-term health effect which includes chronic illnesses which cause serious illness. ${ }^{12}$

The number of neonatal deaths is maximum in these LMICs countries and this can be one of the evidence of the impact of SES. ${ }^{13}$ This increasing neonatal mortality is a serious public health problem and this paper has clearly shown the direct impact of maternal nutrition and underlying factors affecting maternal nutrition and thereby affecting health. ${ }^{14}$

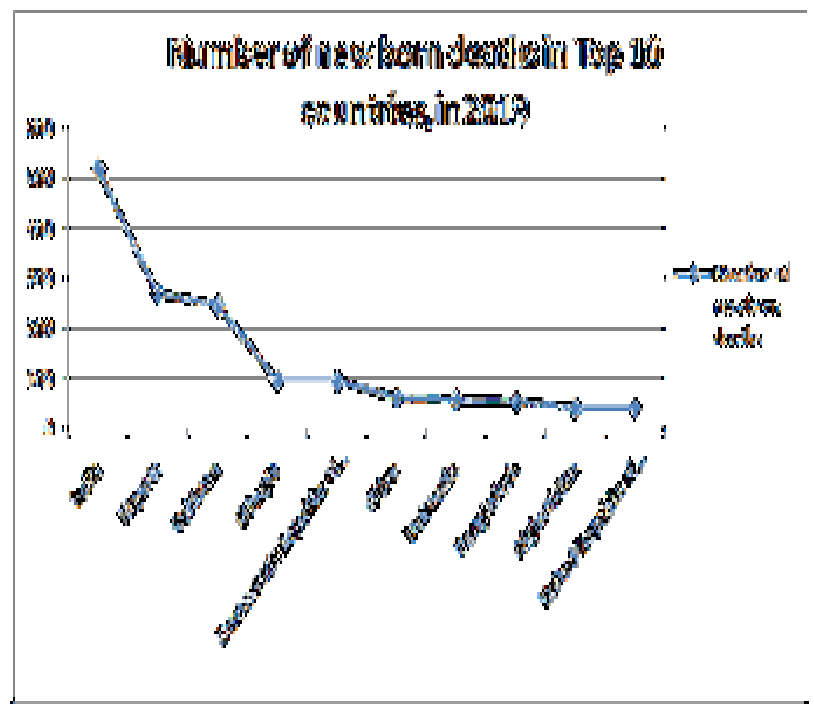

Figure 2: Shows the top 10 countries enlisted by WHO in 2019 with the highest level of newborn deaths. Source: WHO, Author's visualization

Public Health Considerations
The government of India has taken initiatives many times for improvement of the nutritional status of the society which includes large scale distribution of food supplements among young and adults, meal programs, nutritional assessment, etc. The government has also intervened in several programmes for improving the nutritional status of maternal and child health particularly and some of them are: Prophylaxis against nutritional anemia, special nutrition programme, ICDS programme and many more. These initiatives have already been taken by the Government of India, but there is a need for further interventions at various to maintain maternal nutrition and prevent impact on child health. Because Woman at the childbearing stage are also at risk of nutrition deficiency. ${ }^{15}$ Essential public health interventions at ground level is needed which includes: (i) Capacity training \& Understanding of health workers: of the nutrition value and knowledge about the impact of nutrients ${ }^{16}$ (ii) assessment techniques: regular and more precise assessment of the nutritional status of pregnant women ${ }^{17}$ (iii) information, advocacy, Monitoring and Evaluation: awareness and information spreading programme regarding supplementation and nutrients requirement and its importance for child health and regular monitoring and evaluation for the smooth functioning of the system. ${ }^{18}$

\section{Conclusion}

The role of maternal nutrition has remained crucial and is responsible for the adverse birth outcome and child growth. Nutrition can be considered as a modifiable risk factor that has a great public health value. Though there have been studies conducted to check the singlenutrient impact on child health, this primarily involved single pregnancy in a particular time being. This paper talks about the importance of various nutrients and suggests its daily requirement amount, maternal nutrition impact on child health and public health considerations. Though various studies have shown that there is a positive effect of maternal nutrition on child health but the evidence needs to be consistent, for which more in-depth 
studies. There needs to be greater responsibility taken for policy implementation and policymaker need to implement stricter policies based on regular assessment of the nutritional level of pregnant women over a period or in a certain interval of time. Through this paper, a broader view can be given to the nutrition for the evaluation of an existing program or the formation of new programs.

\section{References}

1. Zhang P, Wu J, Xun N. Role of Maternal Nutrition in the Health Outcomes of Mothers and Their Children: A Retrospective Analysis. Med Sci Monit. 2019;25:4430-4437.

2. Duggan MB. Nutritional update: relevance to maternal and child health in East Africa. Afr Health Sci. 2003;3(3):136-143.

3. Ramakrishnan U, Imhoff-Kunsch B, Martorell R. Maternal nutrition interventions to improve maternal, newborn, and child health outcomes. Nestle Nutr Inst Workshop Ser. 2014;78:71-80.

4. Martin-Gronert MS, Ozanne SE. Maternal nutrition during pregnancy and health of the offspring. Biochem Soc Trans. 2006;34(Pt 5):779-782.

5. Fadare O, Amare M, Mavrotas G, Akerele D, Ogunniyi A. Mother's nutrition-related knowledge and child nutrition outcomes: Empirical evidence from Nigeria [published correction appears in PLoS One. 2019 Apr 4;14(4):e0215110]. PLoS One. 2019;14(2):e0212775.

6. Chia AR, Chen LW, Lai JS, et al. Maternal Dietary Patterns and Birth Outcomes: A Systematic Review and Meta-Analysis. Adv Nutr. 2019;10(4):685 -695 .

7. Borge TC, Aase H, Brantsæter AL, Biele $\mathrm{G}$. The importance of maternal diet quality during pregnancy on cognitive and behavioural outcomes in children: a systematic review and meta-analy sis. BMJ Open. $2017 ; 7(9)$ :e01 6777.
8. Saaka M. Relationship between mothers' nutritional knowledge in childcare practices and the growth of children living in impoverished rural communities. J Health Popul Nutr. 2014; 32(2):237-248.

9. Lowensohn RI, Stadler DD, Naze C. Current Concepts of Maternal Nutrition. ObstetGynecolSurv. 2016;71(7):413-426.

10. Imdad A, Bhutta ZA. Maternal nutrition and birth outcomes: the effect of balanced protein-energy supplementation. Paediatr Perinat Epidemiol. 2012;26 Suppl 1:178190.

11. Bamji MS, V S Murthy PV, Williams L, Vardhana Rao MV. Maternal nutritional status \& practices \& perinatal, neonatal mortality in rural Andhra Pradesh, India. Indian J Med Res. 2008;127(1):4451

12. Danielewicz $\mathrm{H}$, Myszczyszyn $\mathrm{G}$, Dębińska A, Myszkal A, Boznański A, Hirnle L. Diet in pregnancy-more than food. Eur J Pediatr. 2017;176(12):15731579.

13. Kavle JA, Landry M. Addressing barriers to maternal nutrition in low- and middleincome countries: A review of the evidence and programme implications. Matern Child Nutr. 2018;14(1):e12508.

14. Metcoff J, Costiloe JP, Crosby W, et al. Maternal nutrition and fetal outcome. Am J Clin Nutr. 1981;34(Suppl 4):708-721.

15. Nnam, N. (2015). Improving maternal nutrition for better pregnancy outcomes. Proceedings of the Nutrition Society, 74(4), 454-459.

16. Abu-Saad K, Fraser D. Maternal Nutrition and Birth Outcomes. Epidemiol Rev. 2010;32(1):5-25.

17. Mason JB, Saldanha LS, Ramakrishnan $\mathrm{U}$, et al. Opportunities for Improving Maternal Nutrition and Birth Outcomes: Synthesis of Country Experiences. Food Nutr Bull. 2012;33(2_suppl1):S104-S137.

18. Victora $\mathrm{CG}$, Barros $\mathrm{FC}$, Assunção $\mathrm{MC}$, Restrepo-Méndez MC, Matijasevich A, 
Martorell R. Scaling up Maternal

Nutrition Programs to Improve Birth

Outcomes: A Review of Implementation
Issues. Food Nutr Bull. 2012;33

(2_supp11):S6-S26. 